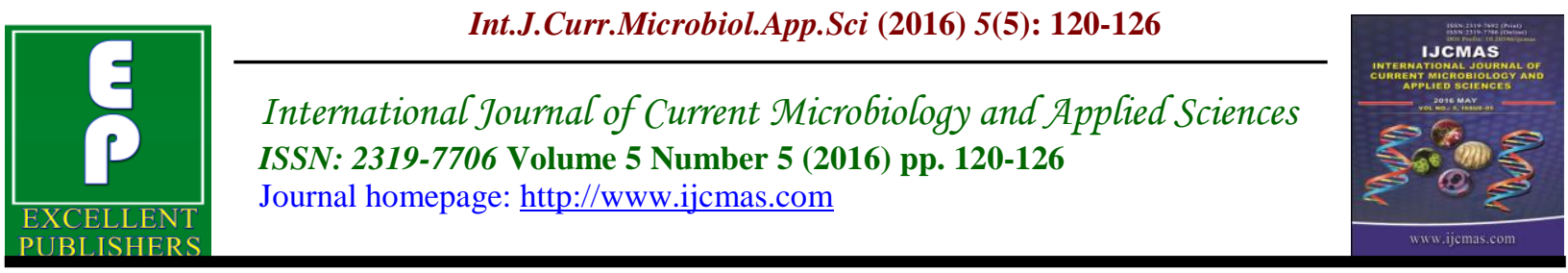

Original Research Article

http://dx.doi.org/10.20546/ijcmas.2016.505.013

\title{
A Study on Aerobic Bacteriological Profile of Sterile Body Fluids
}

\author{
B. Vishalakshi*, Pushpalatha Hanumanthappa and S. Krishna \\ Department of Microbiology, Vijayanagar Institute of Medical Sciences, Ballari, India \\ *Corresponding author
}

\begin{abstract}
A B S T R A C T
This study aims to isolate and identify the aerobic bacterial pathogens of sterile body fluids excluding blood and to determine their susceptibility to various antimicrobial agents. Retrospective observational study was conducted in a tertiary care hospital between January 2014 to December 2015. With universal safety precautions, the sterile body fluids were collected and processed. Identification was done by standard protocols. The antimicrobial susceptibility testing was done for

Keywords

Sterile body fluids; Aerobic bacteriology, Antimicrobial susceptibility testing.

\section{Article Info}

Accepted:

12 April 2016

Available Online:

10 May 2016 the isolates by Kirby Bauer's Disk Diffusion method and MRSA detected and interpreted as per CLSI guidelines. Out of 115 Body fluids samples received for culture and sensitivity, most common received fluid were ascitic fluid $17(14.78 \%)$ followed by synovial fluid 08(6.95\%), pleural fluid 07(6.09\%), fluid from dialysis unit 04(3.5\%),CSF \& hydrocele 01(0.9\%) each and others 77(66.95\%).Among the 115 samples, 30(26.09\%) showed gram stain positive, 17(14.78\%) were culture positive and $98(85.22 \%)$ cultures sterile even after $48 \mathrm{hrs}$ of incubation. Of 17 culture positive samples, $10(85.22 \%)$ samples yielded gram positive cocci (GPC), $04(57.14 \%)$ samples yielded Enterobactericeae and 03(42.86\%) samples grew Non-fermenters. Among gram positive cocci, Staph aureus is the most common with 7(70\%) samples yielding the organism followed by CONS in $02(20 \%)$ samples. Among Enterobactericeae, E.coli is the predominant with 2(50\%) isolates followed by Klebsiella spp (25\%). Among Non-fermenters, we could isolate Pseudomonas aeruginosa in 03(100\%) samples. Most of GPC were sensitive to Vancomycin, gentamicin \& amikacin and least to clindamycin. Methicillin resistance in $S$. aureus was found to be $02(28.57 \%)$. whereas most of GNB were sensitive to aminoglycosides \& least to ampicillin. Low culture positivity may be due to presence of anaerobic; fastidious organisms with lack of enrichment techniques \& prior antibiotic administrations. Regular monitoring of prevalent pathogenic organisms and their sensitivities will aid the clinicians in appropriate selection of antibiotic therapy in absence of a culture report and prevent the development of antibiotic resistance.
\end{abstract}

\section{Introduction}

Body fluids like ascitic, pleural, synovial fluids, cerebrospinal and hydrocele are frequently received samples in the microbiology laboratory for culture in suspected infections. These infections are associated with considerable morbidity and 
mortality. Positive cultures are low because of less number of pathogens as well as prior administration of empirical antibiotics in these samples (Anasua Deb et al., 2014).

Early detection and rapid identification of microorganisms are crucial for the appropriate management (Wiest $\mathrm{R}$ et al., 2011;. van de Beek D et al., 2012; Proulx N et al., 2005). As Availability of such early information helps the clinician to initiate early and more specific treatment and reduced lengths of stay of the patients in the hospital with less adverse effects (Baron EJ et al.,; 1998).There is a need of periodic analysis of the local geographical bacteriological profile and antibiotic susceptibility pattern of organisms isolated and the results need to be communicated to the clinician. So, the present study was undertaken to evaluate aerobic bacteriological profile along with their antibiogram.

\section{Materials and Methods}

\section{Source of Data}

Patients samples from the different speciality wards of the hospital and the outpatient department of VIMS, MCH, Ballari.

\section{Study Type and Duration}

A retrospective observational study was done between January 2014 to December 2015 at VIMS, MCH Ballari. Ethics committe approval was obtained prior to initiation of the study.

\section{Inclusion Criteria}

All sterile body fluids received for aerobic culture and sensitivity from different IPDs \& OPDs in Microbiology Central laboratory of VIMS MCH Hospital, Ballari, India.

\section{Exclusion Criteria}

All blood samples.

\section{Methods}

With universal safety precautions, the sterile body fluids were collected and were subjected for gram stain and culture. The samples were inoculated on Mac Conkey agar and Chocolate agar and incubated at $37^{\circ} \mathrm{C}$ for 48 hours and observed for bacterial growth. Organisms isolated were identified by standard identification procedures (Winn $\mathrm{W}$ et al., 2006) and their antimicrobial susceptibility testing were done for the isolates by Kirby Bauer's Disk Diffusion method and interpreted as per Clinical Laboratory Standard Institution (CLSI) guidelines (CLSI 2013).The cultures were declared sterile if there was no growth on the plates after 48 hours of incubation. Relevant clinical history was collected from each patient for clinical correlation.

Standard antibiotics like, ampicillin (10 $\mathrm{mcg}$ ), vancomycin (30 mcg), ceftriaxone (30 $\mathrm{mcg}$ ), cefotaxime (30 mcg), ceftazidime (30 $\mathrm{mcg}$ ), ciprofloxacin (5mcg), co-trimoxazole $(1.25 / 23.75 \mathrm{mcg})$, gentamycin $(10 \mathrm{mcg})$, amikacin $(30 \mathrm{mcg})$, clindamycin $(2 \mathrm{mcg})$ and erythromycin $(15 \mathrm{mcg})$, Cefoxitin (30 mcg) were tested (HIMEDIA, Mumbai, INDIA) (Betty A et al., 2007).

\section{Detection of Methicillin Resistance}

The methicillin resistance in Staphylococcus spp. was tested by cefoxitin disc $(30 \mu \mathrm{g})$ as documented in Clinical and Laboratory Standard Institute. (Cefoxitin disc diffusion of $\leq 21 \mathrm{~mm}$ for $S$. aureus). The positive culture reports were analysed and percentages and proportions were calculated and analyzed by appropriate statistical analysis. 


\section{Results and Discussion}

A total of 115 Body fluids were processed and shown in flow chart1; of them 17(14.78\%) were ascitic fluids, 08 (6.95\%) synovial fluids, 07 (6.09\%) pleural fluids, 01(0.9\%) CSF, 01(0.9\%) hydrocele fluid, 04(3.5\%) fluids from dialysis unit and 77 (66.95\%) other fluids. Among the 115 samples, $30(26.09 \%)$ showed gram stain positive, $17(14.78 \%)$ were culture positive and $98(85.22 \%)$ cultures sterile even after $48 \mathrm{hrs}$ of incubation. Of 17 culture positive samples, $10(85.22 \%)$ samples yielded gram positive cocci, 04 (57.14\%) samples yielded Enterobactericeae and 03(42.86\%) samples grew Non-fermenters. Among gram positive cocci, Staph aureus is the most common with 7(70\%) samples yielding the organism followed by CONS in 02 (20\%) samples and Strept.spp in 01(10\%) sample. Among Enterobactericeae, E.coli is the predominant with $2(50 \%)$ isolates followed by Klebsiella spp (25\%) and Citrobacter spp (25\%) in each sample. Among Non-fermenters, we could isolate Pseudomonas aeruginosa in 03(100\%) samples as shown in flow chart 1.

Flow chart1 shows Aerobic bacterial isolates of body fluid samples. Figure 1 explains
Specimen type and number of samples processed.

Out of 115 samples, most common received fluid is Ascitic fluid 17(14.78\%) followed by Synovial fluid 08(6.95\%), Pleural fluid $07(6.09 \%)$, fluid from dialysis unit 04(3.5\%), CSF \& hydrocele 01(0.9\%) each and others $77(66.95 \%)$.

Table 1 shows samples profile \& rate of positive culture from different samples. Table 2 explains Sample-wise distribution of the isolates. The pathogens isolated according to the different types of body fluids that were cultured. Figure 2 shows Antibiotic sensitivity pattern of gram positive cocci. Most of GPC were sensitive to Vancomycin, gentamicin, amikacin \& cotrimoxazole. Least sensitive to clindamycin \& Erythromycin. It showed MSSA 5(71.43\%), MRSA 02 (28.57\%).

Figure 3 shows Antibiotic sensitivity pattern of Gram negative bacilli. Most of the GNB were sensitive to Aminoglycosides \& least sensitive to ampicillin. The observation of our study correlates with the other studies across the country.

Table.1 Samples Profile \& Rate of Positive Culture from Different Samples

\begin{tabular}{|l|l|l|}
\hline Sl.no & $\begin{array}{l}\text { Specimen type } \\
\text { (total specimens) }\end{array}$ & $\begin{array}{l}\text { No.of positive specimens } \\
\text { (\% positive) }\end{array}$ \\
\hline 1 & Synovial fluid (08) & $02(11.8 \%)$ \\
\hline 2 & Pleural (07) & $01(5.9 \%)$ \\
\hline 3 & Ascitic fluids(17) & $02(11.8 \%)$ \\
\hline 4 & CSF fluids (01) & $00(00 \%)$ \\
\hline 5 & Hydrocele(01) & $00(00 \%)$ \\
\hline 6 & Dialysis unit(04) & $00(00 \%)$ \\
\hline 7 & Others(77) & $12(70.5 \%)$ \\
\hline & Total $=115$ & $17(100 \%)$ \\
\hline
\end{tabular}


Table.2 Sample-Wise Distribution of the Isolates

\begin{tabular}{|l|l|l|l|l|l|l|}
\hline S1 no & Organism isolated & Synovial & Ascitic & Pleural & Others & Total=17 \\
\hline & Gram positive & & & & & \\
\hline 1 & $\begin{array}{l}\text { Staphylococcus } \\
\text { aureaus }\end{array}$ & - & 02 & 01 & 04 & 07 \\
\hline 2 & CONS & - & - & - & 02 & 02 \\
\hline 3 & Streptococcus spp & 01 & - & - & - & 01 \\
\hline & Enterobacteriaceae & & & & & \\
\hline 4 & E.coli & - & - & - & 02 & 02 \\
\hline 5 & Klebsiella spp & 01 & - & - & - & 01 \\
\hline 6 & Citrobacter spp & - & - & - & 01 & 01 \\
\hline 7 & Non- fermenters & & & & & \\
\hline 7 & $\begin{array}{l}\text { Pseudomonas } \\
\text { aerojinosa }\end{array}$ & - & - & - & 03 & 03 \\
\hline & Total=17 & 02 & 02 & 01 & 12 & 17 \\
\hline
\end{tabular}

Figure.1 Pie Diagram Showing Sample Type and Number of Samples Processed

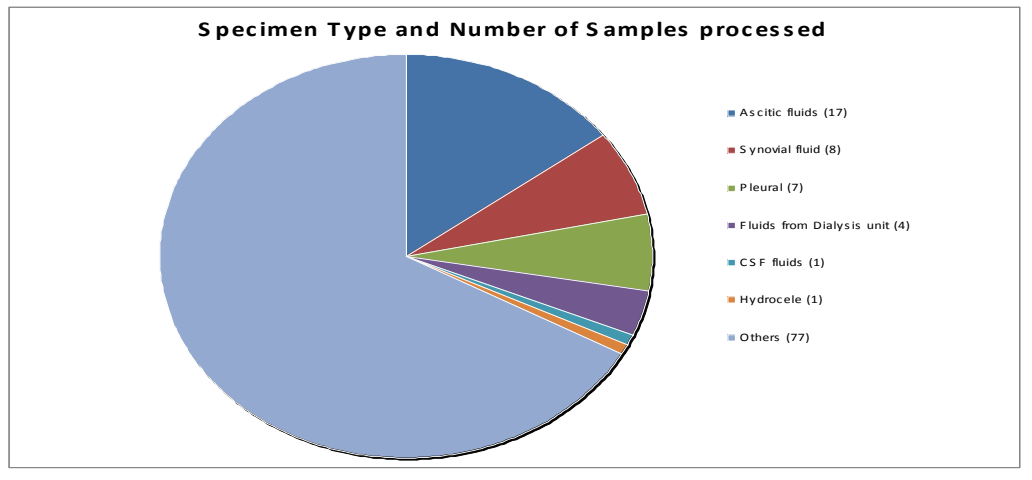

Figure.2 Antibiotic Sensitivity Pattern of Gram Positive Cocci

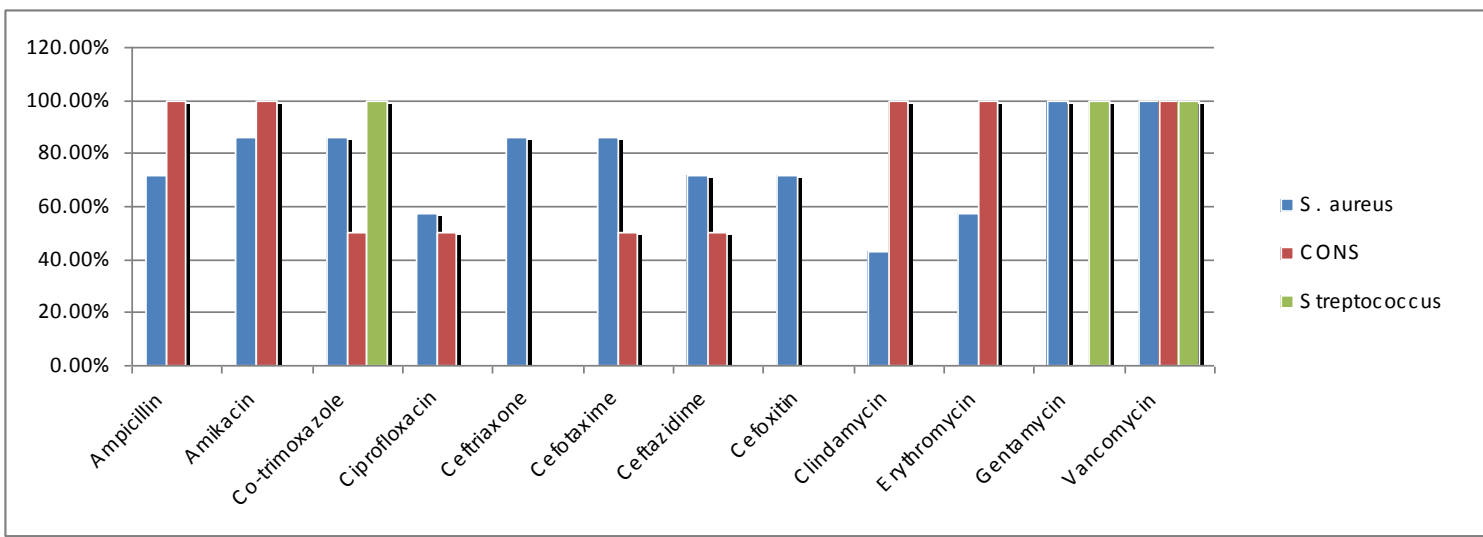


Flow chart.1 Showing 115 Aerobic Bacterial Isolates of Body Fluid Sample

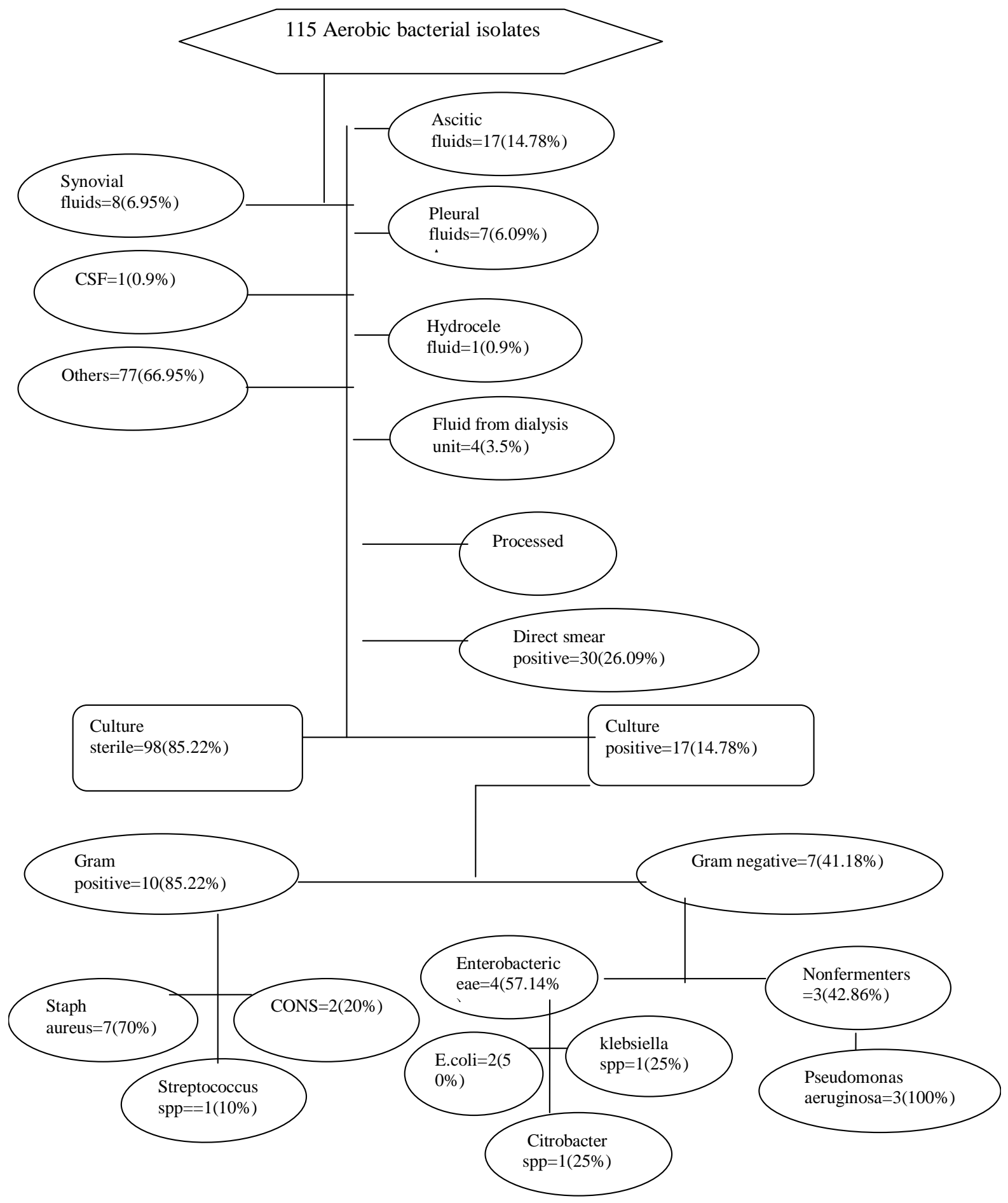


Figure.3 Antibiotic Sensitivity Pattern of Gram Negative Bacilli

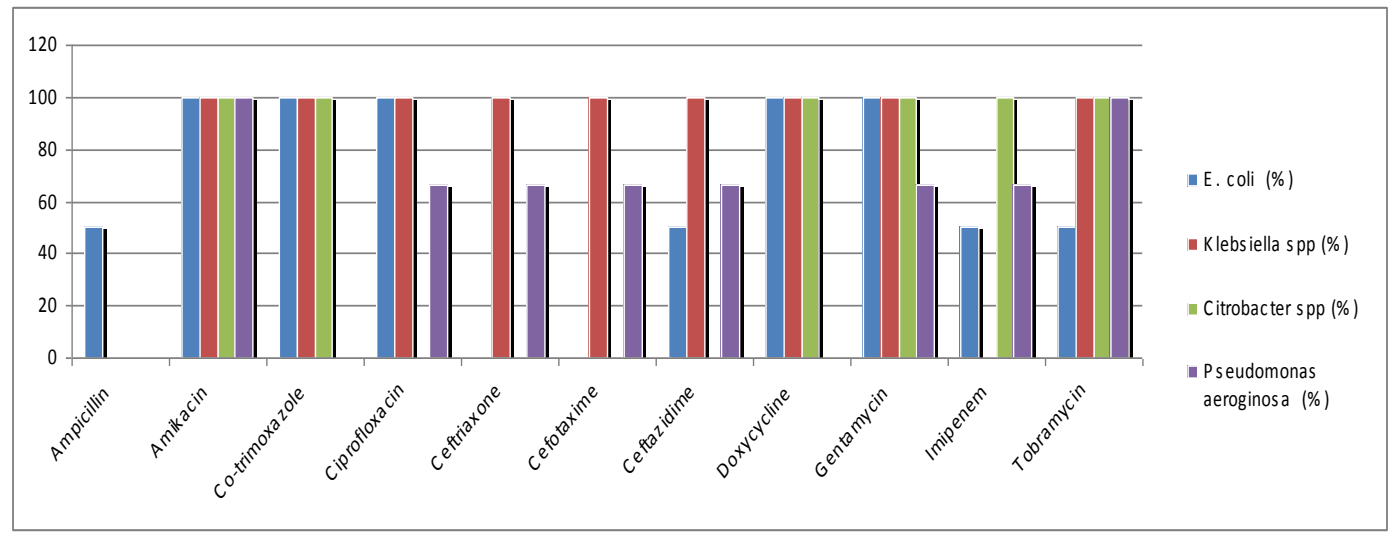

Out of 115 samples in our study, most commonly received fluid is ascitic fluid $17(14.78 \%)$ followed by synovial fluid 08(6.95\%), pleural fluid 07(6.09\%), fluid from dialysis unit $04(3.5 \%)$, CSF \& hydrocele $01(0.9 \%)$ each and others $77(66.95 \%)$. This study correlates with that of Anasua Deb et al., (Anasua Deb et al., 2014) in which a total of 333 Body fluids were processed; 103 of them were ascitic fluids, 71 pleural fluids, $139 \mathrm{CSF}$ and 20 other fluids.

In our study, the culture positivity rate was $14.78 \%$ as it correlates with the study conducted by Paul baurbeau et al., (Paul baurbeau et al., 1998) with rate of $16.68 \%$. In our study, Staph aureus 7(70\%) was the most common pathogen isolated followed by CONS and others. This correlates with Bourbeau et al., (Paul Bourbeau et al., 1998) in which most common organisms were Staphylococcus aureus followed by CONS.

Similar predominance of Gram positive cocci is observed by Yoon et al., (Yoon et al., 2015). Paul et al., (Paul Bourbeau et al., 1998) from Jaipur also mentioned a higher proportion of Gram positive isolates, viz. S. aureus CONS, and Enterococcus, although the distribution of fluid types has not been mentioned in their study.
In our study, most of gram positive cocci were sensitive to vancomycin, gentamicin, amikacin \& cotrimoxazole. This correlates with that of Anasua Deb et al., 2014 in which all the isolates were sensitive to higher drugs like Vancomycin. Further, showed maximum of Methicillin Sensitive Staphylococcus aureus similar to Anasua Deb et al., Most of the Gram negative bacilli were sensitive to aminoglycosides this correlates with Anasua Deb et al., (Anasua Deb et al., 2014)

Our setup being a tertiary care hospital, the critical patients already have prior exposure to antibiotics, which could have resulted in high antimicrobial resistance.

In conclusion, the yield of body fluids cultures is usually very low. Low culture positivity may be due to presence of anaerobic or fastidious organisms with lack of enrichment techniques \& prior antibiotic administration. Regular monitoring of prevalent pathogenic organisms and their sensitivities will aid the clinicians in appropriate selection of antibiotic therapy in absence of a culture report and further prevent the development of antibiotic resistance. 


\section{Acknowledgement}

The authors wish to thank the residents of the Department for helped in the compilation of relevant data.

\section{References}

Anasua Deb, Swati Mudshingkar, Vaishali Dohe, Renu Bharadwaj. 2014. Bacteriology of body fluids with an evaluation of enrichment technique to increase culture - positivity. JEMDS, 3(72): 15230-15238.

BaronM, E.J., Finegold, S.M. 1998. Normally sterile body fluids, Bone and Bone marrow In Bailey and Scott's Diagnostic Microbiology (10 th Edition), 1998. Betty A Forbes, Daniel E Finegold \& Alice S Weissfeld., Mosby publications, NY. 413- 2.

Betty, A., Forbes, Daniel, F., Sahm, Alice, S., Weissfeld. 2007. Bailey \& Scott's Diagnostic $\quad$ Microbiology; $12^{\text {th }}$ edition: p- 210.

Clinical Laboratory Standards Institute (CLSI) guidelines. 2013. Performance standards for antimicrobial susceptibility testing: twentieth informational supplement. CLSI document M1000-S20. Wayne PA: Clinical and Laboratory Standard Institute.
Use of the BacT/Alert Blood Culture System for Culture of Sterile Body Fluids Other than Blood. 1998. J. Clin. Microbiol., p. 3273-3277.

Paul Bourbeau, Julie Riley, Barbara, J., Heiter, Ron Master, Carol Young, Carl Pierson, Proulx, N., Frechette, D., Toye, B., Chan, J., Kravcik, S. 2005. Delays in the administration of antibiotics are associated with mortality from adult acute bacterial meningitis. QJM, 98: 291-298.

Van de Beek, D., Brouwer, M.C., Thwaites G.E., Tunkel, A.R. 2012. Adv. Treatment of Bacterial Meningitis. Lancet, 380:1693-1702.

Wiest, R., Krag, A., Gerbes, A. 2012. Spontaneous bacterial peritonitis: recent guidelines and beyond. Gut, 61: 297-310.

Winn, W., Allen, S., Janda, W., Koneman, E., Procop, G., Schreckenberger, P., et al. Koneman's Color Atlas and Textbook of Diagnostic Microbiology: 6th ed. (Lippincott Williams \& Wilkins, Philadelphia, US) 2006.

Yong-Tao Li, Cheng-Bo Yu, Jian-Rong Huang, Zheng-Ji Qin, Lan-Juan Li. September 2015. Pathogen profile and drug resistance analysis of spontaneous peritonitis in cirrhotic patients. World J. Gastroenterol., 21(36): 10409-10417.

\section{How to cite this article:}

Vishalakshi, B., Pushpalatha Hanumanthappa and Krishna, S. 2016. A Study on Aerobic Bacteriological Profile of Sterile Body Fluids. Int.J.Curr.Microbiol.App.Sci. 5(5): 120-126. doi: http://dx.doi.org/10.20546/ijcmas.2016.505.013 